\title{
Enseñanza práctica en 3D: juicio virtual
}

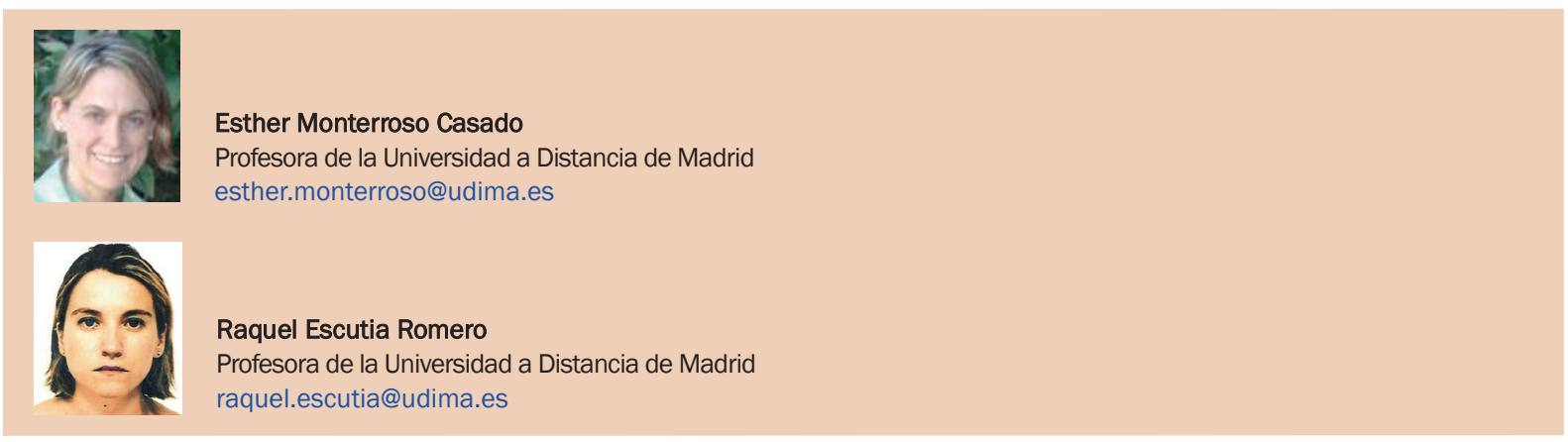

|Fecha presentación: 14/04/2011 | Aceptación: 06/06/2011 |Publicación: 21/06/2011

\begin{abstract}
Resumen
Este artículo describe los resultados de la aplicación de metaversos como herramienta de enseñanza en el ámbito jurídico. La actividad pedagógica realizada se ha llevado a cabo a través de la simulación de un juicio virtual en Second Life. El enfoque dado al ejercicio del derecho en un entorno virtual combinó las siguientes actividades: (1) el análisis jurídico a través de foros de discusión, como una actividad obligatoria previa al juicio. Esta tarea inicial se llevó a cabo a través de la plataforma de aprendizaje asincrónica en 2D Moodle (Aula Judicial); (2) el trabajo colaborativo a través de Google Docs para preparar todos los documentos legales pertinentes (demanda, contestación y tramitación judicial); y (3) la inmersión síncrona en una experiencia 3D de un juicio en Second Life.
\end{abstract}

Palabras clave: Second Life, derecho, simulación juicio, Moodle

\begin{abstract}
Resum
Aquest article descriu els resultats de l'aplicació de metaversos com a eina d'ensenyament en l'àmbit jurídic. L'activitat pedagògica realitzada s'ha dut a terme a través de la simulació d'un judici virtual a Second Life. L'enfocament donat a l'exercici del dret en un entorn virtual ha combinat les següents activitats: (1) I'anàlisi jurídica a través de fòrums de discussió, com una activitat obligatòria prèvia al judici. Aquesta tasca inicial es va dur a terme a través de la plataforma d'aprenentatge asincrònica en 2D Moodle (Aula Judicial); (2) el treball col.laboratiu a través de Google Docs per a preparar tots els documents legals pertinents (demanda, contestació i tramitació judicial); i (3) la immersió síncrona en una experiència 3D d'un judici a Second Life.
\end{abstract}

Paraules clau: Second Life, dret, simulació d'un judici, Moodle

\begin{abstract}
This article describes the results of implementing metaverses as teaching tools in the academic field of law. It is based upon a pedagogical activity that was made possible by the use of a virtual reality court session developed in Second Life. Our approach to virtual law practice combined the following set of activities: (1) legal analysis through discussion forums, as a required pre-trial activity. This initial task was implemented trough the asynchronous learning platform Moodle (Aula Judicial); (2) collaborative work through Google Docs as to prepare all the relevant legal paperwork (claim, statement of defense, judicial proceedings); (3) synchronous inmersion in a Second Life trial experience.
\end{abstract}

Keywords: Second Life, law, mock trial, Moodle 


\section{Introducción}

Las TIC y, en particular, los soportes telemáticos de apoyo a la docencia diseñados para conectar virtualmente al alumno y al profesor, optimizan el proceso de aprendizaje. Dichos soportes facilitan la presentación y el acceso al conocimiento dentro y fuera del aula, promueven la práctica del aprendizaje colaborativo y cooperativo, operan a nivel cognitivo en la edificación de nuevos saberes y destrezas técnicas, y agilizan la retención de conocimientos (López Alonso y Seré 2004). En definitiva, facilitan los aprendizajes y el intercambio informativo en la enseñanza superior.

Dentro de este ámbito, los entornos virtuales en 3D ofrecen un servicio formativo más atractivo para los estudiantes y un vehículo complementario para la enseñanza virtual del profesor universitario, potenciando la adquisición y transmisión de conocimientos a través de un aprendizaje implementado con juegos de rol (role playing), en los distintos ámbitos profesionales. En el mundo jurídico, supone una experiencia interesante e innovadora la simulación de juicios en Second Life, donde los estudiantes pueden representar virtualmente las distintas profesiones jurídicas propias de la actividad forense (abogados demandantes, abogados demandados, procuradores o jueces).

Por ello, el objetivo de este artículo es analizar las implicaciones pedagógicas de la enseñanza inmersiva en mundos virtuales y, en concreto, demostrar cómo Second Life constituye una plataforma que permite potenciar el aprendizaje en un entorno dinámico, visual e interactivo, y un excelente entorno para motivar al alumnado al mismo tiempo que adquirir competencias profesionales.

La hipótesis de partida que plantea nuestro trabajo consiste en verificar la relación existente entre el uso de herramientas docentes telemáticas virtuales y la adquisición de competencias jurídicas por los estudiantes del Grado de Derecho Para ello, se toma como referencia el análisis de los resultados de la experiencia docente que durante los dos últimos años se ha implementado en la Universidad a Distancia de Madrid a través de la simulación de un juicio en Second Life, donde los estudiantes aplican los conocimientos previamente adquiridos en las asignaturas de Derecho a la práctica jurídica de manera similar al ejercicio profesional.

Para el desarrollo de la práctica jurídica en mundos virtuales, se ha compaginado la enseñanza a través del Aula Virtual Moodle (que hemos denominado "Aula Judicial”) donde los estudiantes elaboran, como trabajo previo, una Actividad de Evaluación Continua, consistente en la resolución de un caso práctico; y su escenificación en un juicio dentro de un entorno virtual 3D.

Así, la actividad desarrollada cuenta con dos fases claramente diferenciadas.

La primera -asíncrona en un contexto 2D- se desarrolla en un aula virtual (denominada Aula Judicial), con los recursos que ofrece la plataforma Moodle, entre los que además de las tareas y recursos con los contenidos propios de la temática de la actividad, destaca la utilización de dos herramientas fundamentales: los foros, creados con grupos asociados a los roles que cada estudiante va a interpretar, y el recurso colaborativo de Google Docs, que favorece y fa- cilita el trabajo de redacción del documento que cada grupo habrá de entregar para después representar su rol en el juicio virtual.

La segunda fase -síncrona en un contexto 3D- se desarrolla en el campus virtual en Second Life de la Universidad a Distancia de Madrid (UDIMA) donde tiene lugar el proceso. Los alumnos, a través de su avatar togado, verdaderamente pueden experimentar un alto grado de interacción esencial en el juicio, mediante la representación digital de su propia identidad, adoptando en este caso el rol de los operadores jurídicos (abogado, procurador y juez), en la sala de juicios. De esta forma, se ofrece al estudiante una aproximación al ejercicio del Derecho lo más similar a la realidad procesal, sin barreras espaciales y con la sincronía propia del mundo real.

\section{Las TIC como herramientas de aprendizaje}

El Espacio Europeo de Educación Superior (EESS) pretende favorecer en materia de educación la convergencia europea. El sistema español, a la luz del Real Decreto 1393/2007, de 29 de octubre, por el que se establece la ordenación de las enseñanzas universitarias oficiales, establece como principal objetivo impulsar un cambio en las metodologías docentes, que centra el objetivo en el proceso de aprendizaje del estudiante, en un contexto que se extiende ahora a lo largo de su vida. No se trataría tanto de acentuar lo que se enseña y cómo se enseña, cuanto lo que se aprende y cómo se aprende (Palomino 2009: 299), por lo que resulta preciso incorporar nuevas manera de enfocar la evaluación del proceso de aprendizaje.

Tras la implantación del nuevo sistema, la universidad española en su conjunto (docentes, alumnos y gestores) se enfrenta a grandes desafíos. Además de la modificación de los planes de estudio que repercute en la concepción tradicional de la docencia y de las funciones del profesorado, existen dos particularidades relevantes: el carácter eminentemente práctico de los estudios y el vuelco hacia el ámbito empresarial (Martínez Rojo y González 2009).

En este sentido, Bolonia ha inaugurado un nuevo paradigma educativo en el que el desarrollo de competencias del alumno desplaza la acción directa e inmediata del docente como epicentro de la enseñanza. La mediatez, flexibilidad, apertura del sistema y proactividad ${ }^{1}$ funcionan como ejes centrales del aprendizaje, mientras que el uso de las TIC refuerza, cuando no fundamenta, las destrezas cognoscitivas adquiridas por el estudiante a lo largo de su carrera académica.

La utilización de las TIC en la enseñanza superior, y en particular los soportes telemáticos diseñados para conectar virtualmente al alumno y al profesor, optimizan el proceso de aprendizaje tanto en la educación presencial como en la no presencial (online o a distancia). En otras palabras, las TIC modifican el proceso didáctico, posibilitando el formalismo (el seguimiento de instrucciones derivadas de secuencias muy definidas y precisas), la interactividad (una relación activa y constante con la información en condiciones de reciprocidad y contingencia) o el dinamismo (Badía 2006).

Se pretende así conseguir un modelo pedagógico que se aleje de la enseñanza despersonalizada y masiva, y se centre, por contra, en el propio proceso de aprendizaje del alumno (González Boticario y Gaudioso Vázquez 2003).

${ }^{1}$ El desarrollo tecnológico y la construcción del Espacio Europeo de Educación Superior exigen una adaptación: “estamos ante un cambio de paradigma educativo, pasándose de centrar la atención en la enseñanza (profesor) a centrarse, ahora, en el aprendizaje (alumno), el cambio estructural de los ciclos, grados y créditos y los relacionados con los objetivos de aprendizaje ahora referidos y definidos en términos de competencias son algunas de las razones que obligan a un nuevo planteamiento metodológico" (Tejada et al.2007). 
En este contexto, los entornos virtuales de enseñanza y aprendizaje ofrecen oportunidades ineludibles para el desarrollo de actividades basadas en una metodología de enseñanza-aprendizaje y de trabajo cooperativo. El aprendizaje en red apoyado en la interactividad global, el aprendizaje colaborativo, y el acceso a las actividades y recursos educativos constituye un proceso que se extiende a lo largo de la vida (Harasim et al. 2000). El entorno virtual, pues, facilita estos procesos de cooperación que permitirán enriquecer las propuestas pedagógicas actuales (Guitert et al. 2007).

No olvidemos, además, que existe un número importante de alumnos incapaces de seguir el ritmo de estudios propios de la universidad presencial, bien por razones de índole geográfica, de salud o, simplemente, por no poder conciliar los estudios con la vida familiar o laboral. No hay duda, en este sentido, de que las TIC permiten eliminar estas barreras, contribuyen a una atención más personalizada y permiten una formación que traspasa el horario lectivo (Monterroso 2009) ${ }^{2}$. En este sentido, la telemática aplicada a la enseñanza permite adecuar los currículos del futuro a los retos que plantearán los desarrollos venideros de la sociedad del conocimiento, principio y fin de la filosofía que ha inspirado el Espacio Europeo de Educación Superior.

En este contexto, las universidades españolas han realizado avances importantes en la dotación e implantación de nuevas infraestructuras tecnológicas (Barro Ameneiro, 2004). En los últimos años, el desarrollo tecnológico y las posibilidades de acceso a la información han propiciado que cada vez sean más las universidades que ofrecen a los estudiantes un campus virtual propio o compartido (interuniversitario), como apoyo a la formación presencial (universidad presencial) o como soporte de la formación a distancia (universidad virtual, a distancia: Universitat Oberta de Catalunya o Universidad a Distancia de Madrid). En este modelo, el proceso educacional se caracteriza por la ruptura de la intersección espacio-temporal entre el estudiante y el profesor, así como por una distribución de la información y del contacto de manera virtual.

\section{Entorno 2D Aula Moodle como marco de elabo- ración y apoyo al aprendizaje.}

Para poder desarrollar la actividad de un juicio en $3 \mathrm{D}$ en el entorno virtual de Second Life, se requiere previamente una labor de configuración y preparación de la actividad por parte de docentes y unas tareas previas de aprendizaje por parte de los estudiantes. Los gestores de contenidos educativos (Learning Management Systems) o entornos virtuales de aprendizaje (Virtual Learning Managements) proporcionan una amplia gama de herramientas telemáticas de apoyo a la docencia, ya que el acceso a la plataforma puede realizarse desde cualquier ordenador conectado a la Red.

Uno de estos gestores es Moodle (Modular Object Oriented Dynamic Learning Environment), que constituye desde la última década la aplicación de enseñanza a través de la Web más extendida. Su éxito, como sabemos, se debe principalmente a que se ofrece como software libre y a que ha demostrado mejores resultados pedagógicos que sus competidores como herramientas para cursos web

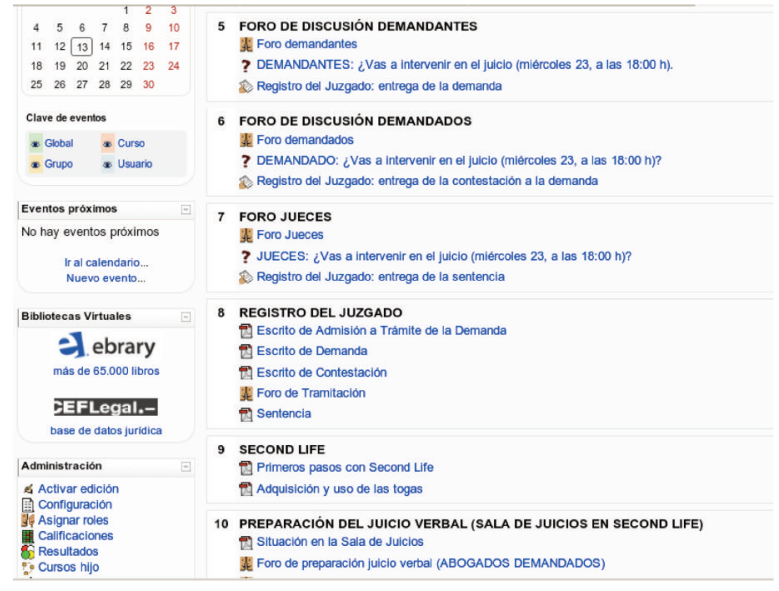

Figura 1. Aula Judicial.

(WebCT). Se trata de un programa diseñado a partir de la teoría del constructivismo social enfocada a la educación en el que el (e-)conocimiento se construye de manera activa y participativa, dado que el alumno se involucra en su propio proceso de aprendizaje. En este sentido, el grupo construye su aprendizaje, unos con otros, compartiendo contenidos y significados de los mismos (Amorós Poveda 2007). Las universidades, y demás centros de educación superior, pueden crear sus propias plataformas Moodle para acceder a los estudios y cursos ofertados, si bien el alumno, tras identificarse como usuario registrado en el sistema, accederá únicamente a las asignaturas en las que esté matriculado.

Las actividades de enseñanza-aprendizaje realizadas a través de Moodle permiten extender virtualmente el aula presencial, presentar los contenidos del curso, enlazar con otros materiales, colaborar en su mantenimiento, redactar y ejecutar cuestionarios, enviar tareas, y proporcionar retroalimentación sobre los contenidos o el desarrollo del curso. Este aprendizaje colaborativo contribuye eficazmente al desarrollo de competencias, permitiendo no sólo la adquisición de las pericias propias de la disciplina, sino también el desarrollo del pensamiento crítico y la reflexión pausada, el hábito para las rutinas de trabajo en equipo, en un entorno de utilización constante de las TIC, así como la búsqueda de datos complementarios o alternativos a los ofrecidos por el docente. Dicho de otro modo, Moodle amplifica tanto las fuentes del conocimiento como la capacidad de transferencia de ese conocimiento (Lee y Tsai 2004).

En el ámbito académico en el que se desarrolla nuestra enseñanza, nos permite potenciar la interacción en consonancia con las características propias del aprendizaje perseguido y las competencias exigidas a los estudiantes de nuestro grado. Al desarrollar la actividad utilizando la plataforma de nuestra universidad (UDIMA), creando en Moodle un Aula Judicial independiente (ver Figura 1), posibilitamos el desarrollo de la actividad práctica del derecho mediante un entorno virtual común y colaborativo de los estudiantes matriculados en nuestras asignaturas y que participan en el juicio.

Para implementar la actividad, contábamos con las herramientas clásicas de Moodle, es decir, los foros, las

\footnotetext{
2 De este modo, el e-learning como sistema de enseñanza: “amplía la formación fuera del horario lectivo de los cursos; permite dedicar sesiones presenciales a los módulos prácticos y relegar a la consulta, fuera del horario lectivo, los temas teóricos con contenidos metódicos; mediante herramientas de comunicación asíncronas se fomenta la reflexión y el debate, contando con tiempo suficiente para definirse un juicio de valores acorde a la temática tratada" (González Martín 2007: 356).
} 
wikis, los talleres y las bases de atos. Para centrar el aprendizaje colaborativo que nuestra disciplina requería al igual que el tipo de actividad a desarrollar, consideramos que las herramientas más adecuadas eran los Foros donde se crearon los espacios propios para la discusión jurídica de cada una de las partes intervinientes en el pleito, así como el uso de la herramienta de Google Docs para la redacción colaborativa de los documentos jurídicos propios del procedimiento.

La herramienta de los Foros permite realizar debates y plantear dudas sobre la asignatura. Ello constituye, sin duda, una de las estrategias fundamentales para guiar el proceso de aprendizaje en la actividad propuesta. Permite esgrimir, por parte de los estudiantes, los argumentos jurídicos que habrían de plasmar con posterioridad en el documento (demanda, contestación a la demanda o expediente de tramitación en la oficina judicial y sentencia) y defender con posterioridad en el juicio virtual en Second Life. Es siempre una oportunidad para potenciar las habilidades de comunicación, lo que forma parte fundamental del futuro patrimonio profesional que cualquier operador jurídico debe dominar.

La configuración de tales Foros por grupos separados facilita el trabajo de cada una de las partes contradictorias, sin que puedan entre ellos conocer el trabajo realizado por cada uno y así mantener el secreto profesional dentro de la actividad.

Para la elaboración de los documentos de la tramitación del pleito, el recurso colaborativo elegido fue Google Docs. Con esta herramienta se puede acceder y editar los trabajos desde cualquier parte, utilizando únicamente un navegador. Además, se comparten los cambios en tiempo real, pudiendo realizar modificaciones conjuntamente, de manera simultánea, y pudiendo todos los autores visualizar esas modificaciones (García Barriocanal y Sicilia Urbán 2009). Al no encontrase en el mismo lugar físicamente todos los estudiantes de manera síncrona realizando la elaboración del documento, la utilidad de esta herramienta reside en que permite un intercambio eficaz de la información entre los miembros del grupo. Se impide, así, que los autores actualicen versiones que no son la última, generando consecuentemente malentendidos y la posibilidad de entregar finalmente una versión mal actualizada.

Para facilitar esta tarea y compartir los documentos de manera colaborativa, creamos un documento para la demanda, otro para la contestación de la demanda, y un último para la sentencia, subiéndolo al espacio virtual de Google Docs y compartiéndolo cada uno de ellos con el grupo de cada rol asignado. De esta manera, se permitió a los estudiantes de nuestras asignaturas modificarlo para plasmar el trabajo colaborativo (a modo de demanda, contestación o sentencia en función de los grupos) que después habría de ser entregado como tarea y valorado con carácter previo a su exposición en el juicio.

\section{Mundos virtuales 3D como potenciadores del aprendizaje: Second Life.}

Un paso más en el desarrollo de la actividad lo constituía la simulación en $3 \mathrm{D}$ del juicio real que ponía en práctica el supuesto resuelto teóricamente.

Los mundos virtuales tridimensionales ofrecen nuevos métodos de aprendizaje en un entorno donde prima la identificación, la elaboración y la experimentación del estudiante. Las principales ventajas que ofrecen los mundos virtuales son: la experiencia que proporcionan, ya que estar e interactuar en un mundo virtual es lo más parecido a los que se puede sentir en el mundo real que simula, así como la creación de un sentido de lugar, ya que los mundos virtuales pueden hacer sentir el estar en un lugar específico, pero sin estar amarrado a un sitio físico. De ahí el crecimiento vertiginoso que han tenido los entornos virtuales en Internet, y que según el último informe de KZero ("Virtual Worlds: 2011 And Beyond”), los mundos virtuales generarán 3.900 millones de dólares a nivel mundial en ingresos, y existirán alrededor de 500 mundos virtuales.

Dentro de estos espacios 3D (Alpha Worlds, Olive, OpenSim, Protosphere, Second Life, Teleplace, Vastpark, Web.alive y Open Wonderland), los más adecuados para la educación conforme a estudios recientes ${ }^{3}$ son OpenSim y Second Life, por la buena capacidad gráfica y herramientas de construcción integradas, que permiten acercarse a un aprendizaje más real que el basado en la lectura. En este sentido, organizaciones como la New Media Consortium (NMC) han promovido el aprendizaje compartido entre educadores y, en un estudio realizado en el 2008 donde se reconocía la utilidad de Second Life para fines educativos, se muestra como resultados más significativos: que los educadores están superando la fase de exploración y ya comienzan a utilizar Second Life para la enseñanza y el aprendizaje; que los educadores están extendiendo sus redes sociales; que tienen más experiencia en Second Life; y que, y quizá sea lo más importante, deseen aprender a crear en Second Life (Lezcano 2010).

Estos ambientes son simulaciones sobre el ordenador que tienen una interfaz en tercera dimensión, para dar a sus usuarios la sensación de inmersión total en todas sus interacciones con el ambiente.

De entre estos entornos de realidad virtual, el elegido ha sido Second Life, una plataforma que permite potenciar el aprendizaje en un entorno inmersivo, dinámico, visual (tridimensional) e interactivo, que constituye un excelente entorno para experimentar con nuevos procesos en la adquisición y transmisión de conocimientos ${ }^{4}$.

Second Life es un mundo virtual multiusuario, 3D y persistente, en el que los usuarios pueden interactuar a través de sus representaciones gráficas llamadas avatares, y pueden conversar en tiempo real usando su propia voz, los gestos de su avatar o la herramienta de chat.

Los usuarios se conectan a Second Life con un software llamado cliente o visor que se comunica con un servidor central. Este cliente muestra el avatar del usuario y la parte

\footnotetext{
${ }^{3}$ El reciente estudio de abril de 2010 realizado por la consultora inglesa Daden Limite sobre la variedad de plataformas de mundos virtuales y el uso de tales mundos para la educación de instituciones del Reino Unido: Southampton Solent Universities, Coventry University, St. George's Hospital en la Universidad de Londres, North Lanarkshire Council, y Birmingham City University, recomienda en un $90 \%$ el uso para usos docentes. Véase http://www.engagedigital.com/2010/05/26/daden-limited-second-life-opensim-best-choices-for-educators/

${ }^{4}$ En este sentido, señala Iribas Rudín que "por su interfaz y sus aspectos más vívidos, visuales y kinestésicos, SL motiva más al alumnado que plataformas meramente bidimensionales y fundamentalmente verbales, como es el caso de WebCT o de Moodle" (Iribas Rudín 2008: 139).

${ }^{5}$ Second Life: http://www.secondlife.com/
} 
del mundo virtual que le rodea, que puede contener paisajes, edificios, otros avatares, etc. A diferencia de otros populares entornos multiusuario como World of Warcraft, Second Life no es un juego por sí solo, ya que el contenido y la narrativa de la historia la construyen sus propios residentes en lugar de Linden Labs, la empresa que proporciona la infraestructura hardware y software de Second Life (Meadows 2008).

Second Life es conocido por aspectos relacionados con el ocio y las empresas, pero también ha llamado la atención de instituciones académicas por su capacidad para ser usado como un recurso adicional a las clases presenciales o como complemento de otras tecnologías 2D como blogs, wikis y foros de discusión (Minocha y Roberts 2008). Una isla de una institución en Second Life puede crear un entorno dedicado al aprendizaje, lo que garantiza un sentido de pertenencia y una finalidad para los estudiantes de la misma forma que un edificio de una universidad presencial. Además la falta de una guía narrativa en Second Life proporciona a los educadores flexibilidad para diseñar espacios de aprendizaje y actividades adaptados a sus necesidades pedagógicas (Reeves y Minocha 2010).

Por ello, se trata de un medio telepresencial, que ofrece la posibilidad de interactuar, mediante un avatar (alter ego virtual), cara a cara (face to face) con otros usuarios (estudiantes y profesores), desterrando uno de los inconvenientes del tradicional e-learning ${ }^{6}$.

La representación de los usuarios mediante avatares proporciona una potente sensación de presencia ${ }^{7}$ en comparación con otras tecnologías web 2D más tradicionales, y el alto nivel de personalización de la apariencia del paisaje y de los avatares es muy atractivo para los usuarios (Rapanotti y Hall 2010).

Por otro lado, investigaciones previas han confirmado que la comunicación síncrona no es importante sólo para la comunicación profesor-estudiante, sino también para establecer relaciones entre los estudiantes. Un entorno que proporciona comunicación síncrona informal entre compañeros de clase respalda las necesidades sociales de los estudiantes (Hrastinski 2006; Petrakou 2010).

Incluso existen previsiones de la industria (Driver y Driver 2009) que afirman que en la próxima década se añadirá a la tecnología web actual una capa inmersiva ${ }^{8}$, como por ejemplo Second Life, que ofrecerá un alto grado de integración, y hará de los mundos virtuales una tecnología dominante para los negocios y la educación.

Se constata así el uso pedagógico de los mundos virtuales, que puede contribuir a disminuir las sensaciones de aislamiento, soledad e incomunicación que pueden experimentar los estudiantes a distancia durante su proceso de aprendizaje. Además, a través del uso pedagógico de los mundos virtuales, se pueden realizar actividades de aprendizaje participativas, constructivas y lo más parecidas posi- bles a cómo serían en el mundo real. Entre las aplicaciones educativas de los mundos virtuales cabe destacar la realización de simulaciones, colaboraciones, juego de roles, trabajar en clases virtuales, construir ambientes colaborativamente, etc.

En este sentido, los entornos virtuales nos ofrecen un servicio formativo interesante para los estudiantes y un vehículo -en gran medida complementario- para la enseñanza virtual por parte del profesor universitario, pues potencian la adquisición y transmisión de conocimientos (Muras 2008), además de posibilitar el aprendizaje a través de juegos de rol (entrenamientos con role playing), en los distintos ámbitos profesionales. Jonathan Richter, investigador del Centro de Tecnologías Avanzadas de la Educación (CATE) en la Universidad de Oregón, ha señalado:

"Terapeutas, soldados, pilotos, abogados, empresarios, médicos, enfermeras y profesores representan roles de la vida real cuando aprenden el contexto y las condiciones particulares de sus profesiones en la universidad o en cursos de formación. Los entornos virtuales multiusuario como Second Life son un medio particularmente adecuado para desarrollar escenarios de role playing que motiven al estudiante al aprendizaje, si se proporciona la mezcla correcta de oportunidad y estructura. De hecho, el juego de roles (role playing) en Second Life y en otros entornos virtuales multiusuario pueden quizá representar una de las más cautivadoras oportunidades educativas para adultos en el siglo XXI”.

En el mundo jurídico, resulta una idea atractiva y muy formativa las simulaciones de juicios, donde los estudiantes experimentan con distintas identidades alternativas: abogados, procuradores, fiscales, jueces o secretarios judiciales. Este entorno virtual en $3 \mathrm{D}$ permite que los alumnos interactúen directamente, y participen en la creación de un argumento que han de defender en un tribunal, frente otro grupo de estudiantes que actúan como parte contraria y ante un tercer grupo que actúa como jueces. Pero Second Life también permite otras aplicaciones, ya que existe gran versatilidad en las actividades que pueden ser realizadas: desarrollar docencia virtual, impartir conferencias o ponencias online, celebrar reuniones, dirigir proyectos de investigación, realizar trabajos en equipo, talleres virtuales, debates, o invitar a intervenir a un colega especialista de otra universidad, eliminando las molestias y los costes económicos derivados de su desplazamiento.

Instituciones y universidades de todo el mundo ya cuentan con una notable presencia en este entorno. A modo de ejemplo, podemos citar, entre las más relevantes, el Instituto Tecnológico de Massachusetts (MIT), las universidades americanas de Harvard, Standford, Columbia, Princeton o Nueva York, las universidades alemanas de Frankfurt o

\footnotetext{
${ }^{6}$ Señalan en este sentido Grané y Muras (2006) que "Los usuarios, sus avatares, sienten que están hablando cara a cara con otros usuarios mediante un chat incluido en el propio entorno. Estas comunicaciones permiten interacciones sociales que no son una simulación de las interacciones humanas, son las interacciones humanas en un nuevo formato".

${ }^{7}$ Se puede definir presencia como un estado de consciencia donde el actor humano tiene la sensación de estar en la ubicación virtual que se le despliega. Es la sensación de estar presente físicamente allí, antes que en su ubicación real. La presencia es el aspecto central de la realidad virtual. Ferré, X., Imbert, R., Martínez, L.A. Pamplona, S, Villalba, E. (2010) Interacción Persona-Ordenador. Madrid. Ediciones CEF.

${ }^{8}$ Se puede definir inmersión como el acto voluntario de obviar todos los estímulos que indican que la experiencia que se presenta no es real y, por tanto, acaparar toda la concentración y atención del usuario. El usuario inmerso en la experiencia virtual se siente parte del entorno virtual como tal (Ferré et al. 2010) ${ }^{9} \mathrm{SL}$ transcript, Special Speaker Series in Second Life, International Society for Technology and Education (2007), recogido por Jarmon, L., Traphagan, T., Mayrath, M., \& Trivedi, A. (2009).
} 


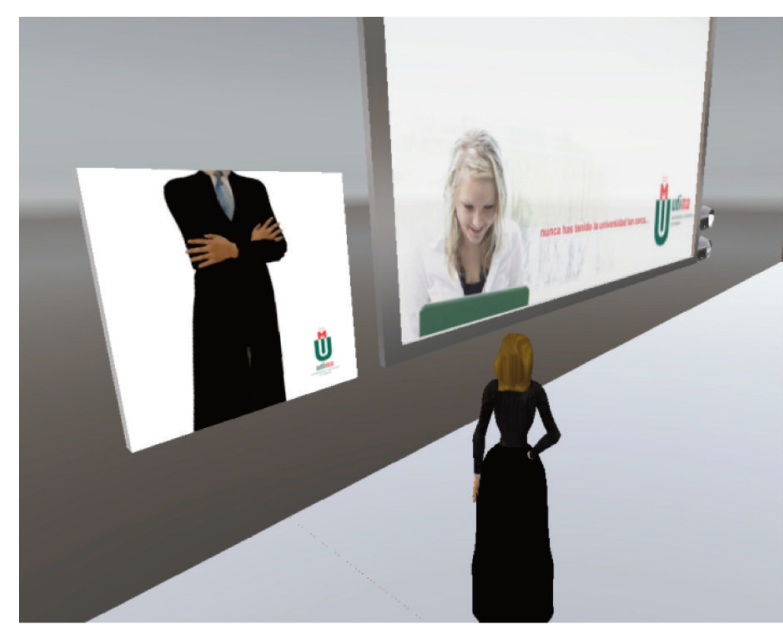

Figura 2. Dispensador de togas.

Hamburgo, la Universidad inglesa de Oxford, Edimburgo o la Open University. En España, las universidades con presencia en Second Life eran inexistentes hasta hace poco más de dos o tres años; sin embargo, en los últimos tiempos algunos centros universitarios han creado una sede corporativa virtual. Entre ellos cabe citar a la Universidad Pública de Navarra, la Escuela Politécnica de la Universidad Autónoma de Madrid, el Instituto de Formación Continua de la Universidad de Barcelona, la Universidad Carlos III, la Escuela Superior de Ingeniería de Vigo, la Universitat Rovira i Virgili, la Universidade da Coruña, la Universidad Miguel Hernández, la Universitat Oberta de Catalunya, o la Universidad a Distancia de Madrid.

Supone pues un nuevo reto en la enseñanza del Derecho que algunas universidades de gran relevancia internacional ya hayan comenzado a utilizar los entornos 3D para entrenar a los futuros abogados, simulando juicios en Second Life. En las universidades españolas, la Universidad a Distancia de Madrid, consciente de que ha comenzando una nueva era en la metodología docente interactiva para el aprendizaje, también ha exportado este sistema como actividad complementaria, ofreciendo a sus alumnos, con carácter voluntario, actividades de aprendizaje en Second Life como complemento a su actividad formativa (Monterroso 2009).

\section{Experiencia docente}

El trabajo desarrollado, como anteriormente se ha mencionado, cuenta con dos fases claramente diferenciadas: la primera, asíncrona y en entorno 2D, que se desarrolla en el aula virtual (denominada Aula Judicial), con los recursos de Moodle, donde se elabora el trabajo de campo y se sigue el procedimiento propio de la oficina judicial, y la segunda síncrona en 3D dentro del campus virtual en Second Life donde se lleva a cabo la puesta en escena de la vista del juicio con los interlocutores propios (abogados, procuradores y jueces). Esta última parte se desarrolla en las instalaciones que posee la UDIMA en Second Life, en la que se recrea una sala de juicios real. En ella los alumnos, a través de su avatar togado (ver Figura 2), pueden experimentar de manera inmersiva la simulación del juicio, mediante la representación digital de su propia identidad, interactuando los roles de los operadores jurídicos el rol de abogado, en la sala de juicios. De esta forma, se ofrece al estudiante una aproximación al ejercicio del Derecho lo más similar a la realidad procesal que vivirán en la realidad práctica de su profesión.

Objetivo y competencias de la actividad

El objetivo de la actividad es la simulación de un juicio verbal aplicando los conocimientos previamente adquiridos en las asignaturas de Derecho Civil: Derecho de Obligaciones y Derecho de los Contratos. Se trata de articular una argumentación jurídica expuesta en una demanda y una contestación, así como la exposición oral de aquélla en el acto del juicio, siendo con posterioridad publicada la sentencia que resuelve el pleito.

Esta actividad pretende que el estudiante adquiera la competencia de redactar escritos jurídicos relacionados con las asignaturas como estímulo de la creatividad personal, maneje tanto el lenguaje técnico jurídico como las bases de datos y sistemas informáticos para la búsqueda de información, ejercite la capacidad de argumentar y debatir acerca de las normas e instituciones civiles en el seno de equipos o grupos de trabajo, y su plasmación práctica en un proceso judicial, conforme a lo establecido en las normas procesales.

La utilización y adopción de espacios virtuales en el aprendizaje tiene una implicación sobre dónde, qué y cómo aprender. El protagonista es el estudiante, que deja de tener una actitud pasiva para interactuar con el profesor y el resto de compañeros actuando conforme a un rol predeterminado y elegido previamente.

En la práctica llevada a cabo no se ha querido descuidar ningún detalle, por lo que se ha exigido a los letrados el uso de la preceptiva toga durante la vista.

\section{Desarrollo e implementación de la actividad}

Para el desarrollo del caso, los estudiantes debían adoptar un papel en función de la posición que debían defender. Unos adoptaron el rol de demandantes, otros demandados y un tercer grupo el de jueces, todos estos roles elegidos a través de una consulta previa en el Aula Judicial. Con el fin de que intercambiaran sus opiniones sobre el supuesto de hecho, se abrió un foro de debate configurado por grupos separados en el Aula Judicial, donde los estudiantes pudieron comunicarse y reflexionar sobre las posibles argumentaciones para la elaboración de la demanda y contestación.

Para efectuar esta primera fase fue necesaria una previa labor de investigación por parte del estudiante, ya que debían realizar un análisis de las cuestiones jurídicas planteadas y recoger la regulación legal sobre la institución jurídica que era objeto de debate. Seguidamente, debieron analizar los aspectos fundamentales de la doctrina y la jurisprudencia relacionada. Algunos de estos recursos fueron facilitados, directa o indirectamente, en el propio Aula Judicial.

A través de un Google Docs, los estudiantes realizaron una demanda conjunta que fue enviada a través del buzón de entrega de la tarea, habilitado en el sistema Moodle, en el Aula Judicial, con el fin de que pudieran ser objeto de calificación. Dicha demanda fue admitida a trámite por el grupo que representaba al órgano judicial a través del correspondiente escrito procesal. Posteriormente, se dio traslado de la misma a los estudiantes que asumieron el rol de demandados, que realizaron la correspondiente contestación a la demanda para exponerla oralmente en el acto de la vista del juicio verbal. 


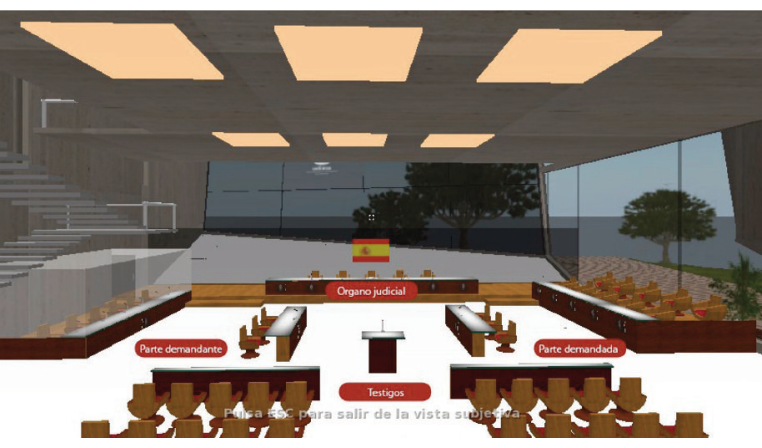

Figura 3. Imagen del tutorial d ela situación en la sala de juicios.

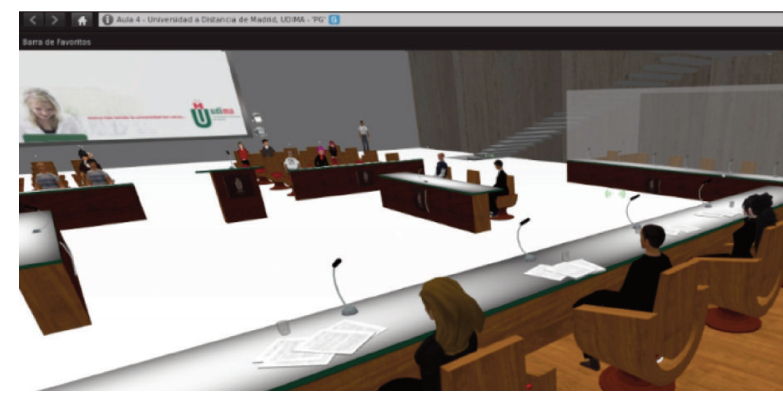

Figura 4. Vista general del pleito en la sala de juicios de la UDIMA en Second Life

Las etapas progresivas que debían ser realizadas por las partes intervinientes eran las siguientes:

\section{a) Parte demandante y demandada}

- Etapa I. Estudio y análisis individual.

Análisis del caso, consulta de legislación y doctrina adicional a la incluida en las unidades didácticas.

- Etapa II. Investigación en grupos separados a través del foro de discusión. Profundización colectiva.

- Etapa III. Elaboración y envío de una demanda individual o contestación a la misma, que sería evaluada por el profesor.

- Etapa IV. Elaboración a través de Google Docs, de manera colaborativa, de una demanda por los demandantes, que sería interpuesta para su contestación por los demandados, mediante el mismo procedimiento. Ambos documentos servirían de documento de apoyo durante el juicio.

- Etapa V: Preparación del juicio verbal a desarrollar en la sala de juicios de Second Life.

- Etapa VI. (opcional) Defensa de la posición de demandantes o demandados en el entorno virtual Second Life.

\begin{tabular}{|c|c|}
\hline Debilidades & Amenazas \\
\hline $\begin{array}{l}\text { La participación ha de ser voluntaria por ser una actividad } \\
\text { sincrónica en una universidad a distancia. }\end{array}$ & $\begin{array}{l}\text { Los problemas técnicos que puedan surgir por la necesidad } \\
\text { de un equipo adecuado, así como de un ancho de banda sufi- } \\
\text { ciente. }\end{array}$ \\
\hline Fortalezas & Oportunidades \\
\hline $\begin{array}{l}\text { Presencialidad e inmersión en mundos } 3 \mathrm{D} \text {. } \\
\text { Mayor grado de interacción entre profesor y alumno, así } \\
\text { como entre los alumnos. } \\
\text { Role playing que estimula y hace más atractivo el aprendi- } \\
\text { zaje. } \\
\text { Potenciación del aprendizaje mediante experiencias prácticas. }\end{array}$ & $\begin{array}{l}\text { Respaldo legislativo en el impulso de nuevos procesos de } \\
\text { aprendizaje en materia de educación superior. } \\
\text { Desarrollo de nuevos métodos pedagógicos innovadores en una } \\
\text { universidad española (UDIMA) pionera en esta experiencia. }\end{array}$ \\
\hline
\end{tabular}

Tabla 1. Análisis DAFO del juicio virtual en Second Life

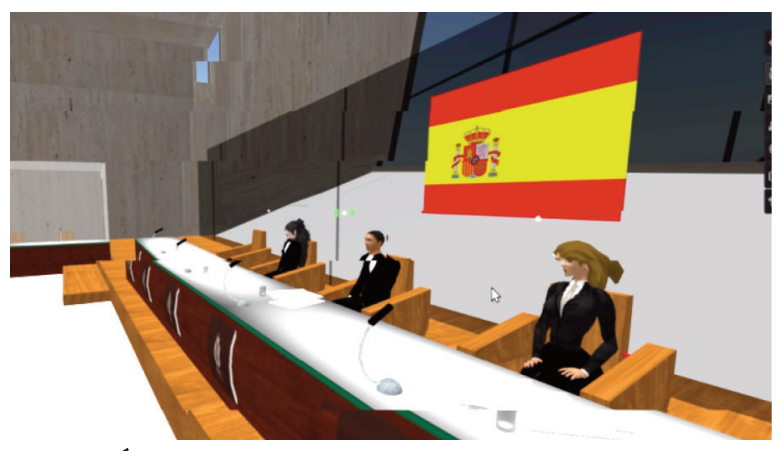

Figura 5. Órgano judicial en el desarrollo del pleito.

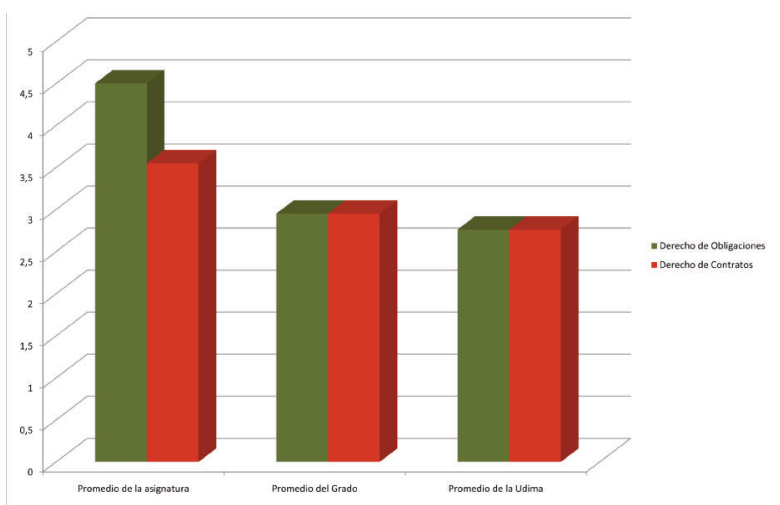

Gráfico 1. Resultados.

b) Jueces

- Etapa I. Estudio y análisis individual de la demanda y contestación a la demanda presentada.

- Etapa II. Investigación en subgrupo separado a través del foro de discusión. Profundización colectiva.

- Etapa III. (Opcional) Desempeño del rol de juez en el entorno virtual Second Life.

- Etapa IV. Elaboración y envío de la sentencia.

Visualización de la actividad, valoración de los estudiantes $y$ resultados.

Las Figuras 3, 4 y 5 son varias imágenes ilustrativas de la actividad didáctica desarrollada en Second Life.

Valoración de la actividad por los estudiantes

Finalizado el curso (segundo semestre 2009/2010) y la actividad, se realizó un encuesta a los estudiantes de ambas asignaturas (Derecho de Obligaciones y Derecho de los Contratos) donde se les preguntaba expresamente sobre la valoración de la herramienta de Second Life utilizada en la Simulación. Los resultados son, como puede comprobarse en el Gráfico 1, altamente satisfactorios. 
Se muestra una comparación de la valoración media de la herramienta Second Life en las asignaturas donde se realizó la simulación del juicio (Derecho de Obligaciones y Contratos), el promedio de la herramienta en todas las asignaturas del Grado de Derecho y, por último, el promedio en todos los Grados de la universidad (UDIMA). A la vista del gráfico se puede concluir que la valoración en dichas asignaturas es aproximadamente un 50\% mayor que en el resto de asignaturas del Grado y de la universidad. Todo ello es debido a que la actividad desarrollada aprovecha todo el potencial de Second Life y, en especial, las ventajas de role-playing.

Por último quisiéramos -antes de concluir- cerrar la exposición con el análisis DAFO sobre la experiencia realizada.

\section{Conclusiones}

Moodle y los entornos virtuales -en especial Second Lifeofrecen un servicio formativo interesante para los estudiantes y un medio útil en la enseñanza virtual universitaria, ya que además de potenciar la adquisición y transmisión de conocimientos, posibilita el aprendizaje a través de juegos de rol en los distintos ámbitos profesionales. En el Grado de Derecho es posible llevar a cabo una estrategia educativa mucho más participativa y adecuada para la adquisición de las competencias propias que deberán utilizar en su futura actividad profesional, tales como la resolución de casos prácticos o la redacción de escritos ejercitando la capacidad de argumentar o el manejo de bases de datos y sistemas informáticos para la búsqueda de información. Además, mediante el desarrollo de esta atractiva actividad, la simulación de juicios en Second Life, los estudiantes pueden interpretar las distintas identidades de los operadores jurídicos en un entorno inmersivo.

La Universidad a Distancia de Madrid ha sido pionera en ofrecer esta actividad en nuestro país. En concreto, en el Grado de Derecho, como complemento de la formación del alumno, se realiza la simulación de un juicio en el entorno virtual de Second Life. En el ámbito del Derecho civil, se ha implementado esta actividad a través de dos fases claramente diferenciadas tanto en contenido como en metodología: entorno 2D Moodle y entorno 3D Second Life.

A través de esta articulación, la actividad deja de consistir únicamente en dar con la solución final de caso con una aplicación teórica de los conocimientos del Derecho Civil (aplicando, en este caso, el Código Civil, la Ley de Arrendamientos Urbanos y la Ley de Enjuiciamiento Civil), ya que lo que se trata es de construir una solución al problema con argumentaciones orales y escritas, lo que responde a la modalidad de aprendizaje autodirigido orientado conforme al EESS, englobando tanto la adquisición de conocimientos como la aplicación práctica de los mismos, ya que se adquieren las competencias, destrezas y habilidades propias del trabajo individual y del colaborativo, y del ejercicio práctico del Derecho de una forma atractiva, que ha sido acogida por los estudiantes con satisfacción según los resultados obtenidos.

Este modelo educativo implica un aprendizaje activo, diseñado y aplicado desde la práctica, y que permite evaluar el grado de solidez que cabe atribuir a una argumentación, aprovechar y desarrollar de forma efectiva las cualidades y capacidades de los estudiantes y, en definitiva, utilizar efectivamente la tecnología.

\section{Bibliografía}

Amorós Poveda, Lucía (2007): Moodle como recurso didáctico. EDUTEC 2007. Inclusión digital en la Educación Superior: Desafíos y oportunidades en la
Sociedad de la Información. Buenos Aires, 23-26 de octubre de 2007 http://www.utn.edu.ar/aprobedutec07/docs/150.pdf. Fecha de consulta: 15.04.2011.

Areitio, Gloria; Areitio, Ana (2002). Nuevas formas de trabajo para el docente frente a los nuevos modelos de enseñanza universitaria. Scripta Nova. Revista Electrónica de Geografia y Ciências Soclaes, Vol. VI, $\mathrm{n}^{0} 119$ http://www.ub.es/geocrit/sn/sn119138.htm. Fecha de consulta: 12.04.2011.

Badia, Antoni (2006). Ayuda al aprendizaje con tecnología en la educación superior. Revista de Universidad y Sociedad del Conocimiento, vo.2, $\mathrm{n}^{\mathrm{o}} 2$, http://www.uoc.edu/rusc/3/2/dt/esp/badia.pdf Barro Ameneiro, Santiago (2004): Las Tecnologías de la Información y las Comunicaciones en el Sistema Universitario Español. Madrid: CRUE.

Driver, E. and Driver, S. (2009). Immersive internet business value study. Immersive Internet Analyst Report Series 2, ThinkBalm.

Ferré, Xavier et al. (2010). Interacción Persona-Ordenador. Madrid. Ediciones CEF.

García Barriocanal, E.; Sicilia Urbán, M.A. (2009). Aprendizaje y Tecnologías de la Información y la Comunicación. Madrid: CEF.

González Boticario, Jesús.; Gaudioso Vázquez, Elena (2003). Sistemas interactivos de enseñanza aprendizaje. Madrid: Sanz y Torres.

González Martín, C. (2007): Campus Virtual para las titulaciones en modalidad presencial, semipresencial y/o distancia. En Ana Landeta (Ed.), Buenas Prácticas de E-Learning, Madrid: Anced.

Grané, Mariona; Muras, Miguel Ángel (2006) Second Life, entorno virtual, aprendizaje real. Tercer Congreso online - Observatorio para la Cibersociedad, http://www.cibersociedad.net/congres2006/gts/comu nicacio.php?llengua $=$ es\&id $=851$ Fecha de consulta: 14.03.2011.

Hrastinski, Stefan. (2006). Introducing an informal synchronous medium in a distance learning course: How is participation affected? The Internet and Higher Education, 9, pp. 117-131.

Iglesias, María del Angel. (2009): Simulación de juicios: los Law Moot Court. En R. Palomino R. y J. RodríguezArana (Dirs.): Enseñar Derecho en el siglo XXI. Pamplona: Aranzadi, pp. 273-281..

Informe de KZero, "Virtual Worlds: 2011 And Beyond", http://www.tecnolives.com/mas-de-500-millones-demundos-virtuales-para-2011-segun-kzero/. Fecha de consulta: 25.05.2011.

Iribas Rudín, Ana Eva (2008): Enseñanza virtual en Second Life: una opción online animada para las universidades y las artes. En IV Jornadas Campus Virtual, Madrid: UCM, pp.125-142

Jarmon, Leslie; Traphagan, Tomoko; Mayrath, Miche; Trivedi, Avani (2009). Virtual world teaching, experiential learning, and assessment: An interdisciplinary communication course in Second Life. Computers \& Education, 53 (1), pp.169-182. http://www.sciencedirect.com/science/article/pii/S 0360131509000141

Lee, C. I.; Tsai, F. (2004): Internet project-based learning environment: the effects on thinking styles on learning transfer, Journal of Computer Assisted Learning, 20, 31-39. 
Lezcano, L. (2010), E- Learning y Second Life. En Ana Landeta (Ed.), Nuevas tendencias de e-learning y actividades didácticas innovadoras. Madrid: CEF.

López Alonso, Covadonga; Seré, Arlette (2004): Entornos formativos en el ciberespacio: las plataformas educativas. Español Actual. Revista de español vivo, $\mathrm{n}^{\mathrm{O}} 82$, pp.9-20.

Martínez Rojo, Estrella; González Fernández, Ana María (2009) Renovación, Innovación y TIC en el EEES. Icono14. Revista de Comunicación y Nuevas Tecnologías, $\mathrm{n}^{\mathrm{o}}$ 14, pp.50-63. http://www.icono14.net/revista/num14/o3_icono14_estrellamartinez.pdf Fecha de consulta: 22.04.2011.

Meadows, Mark Stephen (2008), Avatar: The culture and consequences of having a Second Life. California: New Riders.

Miguel Díaz, Mario De (2006) Metodologías de enseñanza y aprendizaje para el desarrollo de competencias. Orientaciones para el profesorado universitario ante el Espacio Europeo de Educación Superior. Madrid: Alianza.

Minocha, S.; Roberts, D. (2008), Laying the groundwork for socialisation and knowledge construction within $3 \mathrm{D}$ virtual worlds. ALT-J: Research in Learning Technology, Vol. 16, nº 3, pp. 181-196.

Monterroso Casado, E (2009). Los entornos virtuales de aprendizaje: una aplicación práctica. En R. Palomino y J. Rodríguez-Arana J (Dirs.): Enseñar Derecho en el siglo XXI). Pamplona: Aranzadi, pp. 317-329.
Muras, Miguel Ángel (2008): Experiencia de formación en Second Life. Learning Review, 3 http://www.learningreview.es/component/content/978?task=view Fecha de consulta: 23.05.2009.

Palomino Lozano, Rafael (2007): El campus virtual en la enseñanza del Derecho: elaboración de dictámenes e informes. III Jornadas Campus Virtual. Madrid: UCM.

Petrakou, A. (2010). Interacting through avatars: Virtual worlds as a context for online education. Computers \& Education, 54 (4), pp.1.020-1.027.

Rapanotti, Lucia; Hall, Jon (2010). Lessons learned in developing a Second Life educational environment. Proceedings of the 2nd International Conference on Computer Supported Education, 7-10 April 2010, Valencia, Spain

Reeves, A.J.; Minocha, S. (2010). Relating Pedagogical and Learning Space Designs in Second Life. En Amelia Cheney y Robert Sanders, (eds.), Teaching and Learning in $3 D$ Immersive Worlds: Pedagogical Models and Constructivist Approaches. USA: IGI Global.

Tejada José; Navío, Antonio; Ruiz Bueno, Carmen (2007): La didáctica en un entorno virtual interuniversitario: experimentación de ECTS apoyados en TIC, Revista Pixel-Bit. Revista de Medios y Educación, 30. http://www.sav.us.es/pixelbit/pixelbit/articulos/n3o/ n3oart/art303.htm Fecha de consulta: 14.03.2011. 